\title{
THE HOMOTOPY TYPE OF THE SPACE OF GRADIENT VECTOR FIELDS ON THE TWO-DIMENSIONAL DISC
}

\author{
PIOTR BARTŁOMIEJCZYK \\ Institute of Mathematics, University of Gdańsk, Wita Stwosza 57, 80-952 Gdańsk, Poland \\ e-mail: pb@mat.ug.edu.pl,web page: http://pb.mat.ug.edu.pl \\ and PIOTR NOWAK-PRZYGODZKI \\ Faculty of Applied Physics and Mathematics, Gdańsk University of Technology, Gabriela Narutowicza 11/12, \\ 80-233 Gdańsk, Poland \\ e-mail:piotrnp@wp.pl
}

(Received 17 June 2011; revised 28 December 2011; accepted 3 January 2012; first published online 30 March 2012)

\begin{abstract}
We prove that the inclusion of the space of gradient vector fields into the space of all vector fields on $D^{2}$ non-vanishing in $S^{1}$ is a homotopy equivalence.
\end{abstract}

2010 Mathematics Subject Classification. Primary: 55Q05, Secondary: 37B35.

1. Introduction. The main goal of our paper is to prove some version of the well-known Parusiński theorem (see [5]), which says that if two gradient vector fields on the unit disc $D^{n}$ and non-vanishing in $S^{n-1}$ are homotopic, then they are also gradient homotopic. In other words, the inclusion of the space of gradient vector fields in the space of all vector fields on $D^{n}$ non-vanishing in $S^{n-1}$ induces the bijection between the sets of path-components of these function spaces. In this paper we restrict ourselves to the two-dimensional case, but we also strengthen the mentioned result via showing that the above inclusion is a homotopy equivalence (both spaces are homotopy equivalent to $S^{1}$ ). Precisely, this was partially proved in [5] using the argument of deformation retraction, but this method fails (at least in that form) in the case of identity components. For this reason we wish to investigate this (more difficult in our opinion) case here.

It may be worth pointing out that even though Parusiński's result does not hold for equivariant maps, his techniques may be still used to study homotopy classes of gradient equivariant maps (see [1-4]).

The organisation of the paper is as follows. Section 2 contains some preliminaries. Section 3 presents the so-called Parusiński's Trick, which allows to replace gradient vector fields on a two-dimensional disc by pairs of functions on $S^{1}$ without common zeros and one of which has integral over $S^{1}$ equal to zero. In Section 4 our main results are stated. These results are proved in Section 5.

2. Preliminaries. Let $I=[0,1]$. We will denote by $D^{2}$ the unit disc in $\mathbb{R}^{2}$ and by $S^{1}$ its boundary. We often denote a point in $S^{1}$ by its angle $\theta \in[0,2 \pi]$. Similarly,

The second author was supported by the Ministry of Science and Higher Education, Poland, grant no. NN201373236. 
maps on $S^{1}$ will be identified with $2 \pi$-periodic maps on $[0,2 \pi]$. If $\varphi$ is such a map, then $\operatorname{Im} \varphi:=\varphi([0,2 \pi])$. We will consider continuous maps on $D^{2}$ with no zeros on its boundary and their homotopies, i.e. continuous maps $h: D^{2} \times I \rightarrow \mathbb{R}^{2}$ non-vanishing on $S^{1} \times I$. It is well known that homotopy classes of such maps are classified by the topological degree. Recall that a map $f$ is called gradient if there is a $C^{1}$-function $\varphi: D^{2} \rightarrow \mathbb{R}$ such that $f=\nabla \varphi$. Similarly, we say that a homotopy $h: D^{2} \times I \rightarrow \mathbb{R}^{2}$ is gradient if $h(x, t)=\nabla_{x} \chi(x, t)$ for some continuous function $\chi$ that is $C^{1}$ with respect to $x$. Of course, we still assume that $f$ (resp. $h$ ) has no zeros on $S^{1}$ (resp. $S^{1} \times I$ ).

3. Parusiński's trick in the plane. Let $V$ denote the space of all continuous maps on the unit disc in $\mathbb{R}^{2}$ non-vanishing on its boundary, i.e.

$$
V=C^{0}\left(D^{2}, S^{1} ; \mathbb{R}^{2}, \mathbb{R}^{2} \backslash\{0\}\right)
$$

and $G$ denote the subspace of $V$ consisting of gradient maps, i.e. $G=\{f \in V \mid$ $f$ is gradient $\}$.

We will also need the following mapping spaces:

$$
\begin{aligned}
& \mathcal{V}:=C^{0}\left(S^{1} ; \mathbb{R}^{2} \backslash\{0\}\right), \\
& \mathcal{G}:=\left\{(f, g) \in \mathcal{V} \mid \exists \eta: S^{1} \stackrel{C^{1}}{\rightarrow} \mathbb{R} \text { such that } f=\eta^{\prime}\right\} .
\end{aligned}
$$

All the above function spaces are equipped with natural compact-open topology. It is well known that path-components (homotopy classes) of $V$ and $\mathcal{V}$ are classified by the topological degree. Let us denote the path-component consisting of maps of degree $k$ by $V_{k}$ and $\mathcal{V}_{k}$. Moreover, let $G_{k}=G \cap V_{k}$ and $\mathcal{G}_{k}=\mathcal{G} \cap \mathcal{V}_{k}$.

We will make use of the following easy consequence of the above definitions.

Proposition 3.1. If $(f, g) \in \mathcal{V}$, then $(f, g) \in \mathcal{G}$ iff $\int_{0}^{2 \pi} f \mathrm{~d} s=0$.

Let $\vec{\tau}$ (resp. $\vec{v}$ ) denote the unit tangent (resp. normal) vector field on $S^{1}$. Consider the following natural mapping, $\mathcal{P}: V_{k} \rightarrow \mathcal{V}_{k-1}$

$$
\mathcal{P}(v)=(f, g),
$$

where $v\left\lceil S^{1}=f \cdot \vec{\tau}+g \cdot \vec{v}\right.$. Observe that for $v \in G$ we have

$$
\mathcal{P}(v)=\mathcal{P}(\nabla \varphi)=\left.\left(\frac{\partial \varphi}{\partial \theta}, \frac{\partial \varphi}{\partial r}\right)\right|_{S^{1}},
$$

in particular $\mathcal{P}(v) \in \mathcal{G}$. The following result can be found in [5, Lemma 1].

THEOREM 3.2 (Parusiński's trick in the plane). The following diagram commutes

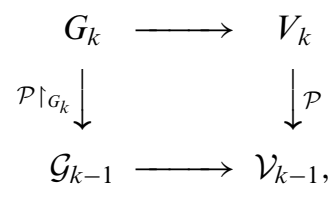

where the horizontal arrows are inclusions and the vertical arrows are homotopy equivalences. 
4. Main result. Let us formulate the main result of this paper.

Main Theorem. The horizontal inclusions in diagram (3.1) are homotopy equivalences.

From the commutativity of the diagram, it is enough to show that for all $k \in \mathbb{Z}$, the inclusion $i: \mathcal{G}_{k} \rightarrow \mathcal{V}_{k}$ is a homotopy equivalence. In fact, for $k \neq 0$ this was proved by Parusiński and we simply repeat his short and elegant argument. But for $k=0$ the proof requires a modified approach.

Maps $v \in \mathcal{V}$ will be often written in polar coordinates as $v(\alpha)=(\theta(\alpha), r(\alpha))$ or $v=(\theta, r)$ for short. We use the standard transformation from polar coordinates to Cartesian ones $\Pi(\theta, r):=(r \cos \theta, r \sin \theta)$, which is a local diffeomorphism. We will need the following simple observation.

Proposition 4.1. The evaluation map $e: \mathcal{V}_{k} \rightarrow S^{1}$, defined by $e(v)=\theta(0)$ for $v=$ $(\theta, r)$, is a homotopy equivalence.

Proof. Since the inclusion $\operatorname{Map}\left(S^{1}, S^{1}\right) \hookrightarrow \mathcal{V}$ is a homotopy equivalence, it is enough to use the 'lifting' argument or to apply the homotopy sequence of evaluation fibration.

Observe that our Main Theorem is an immediate consequence of the following result.

Main Lemma. In the following commutative diagram

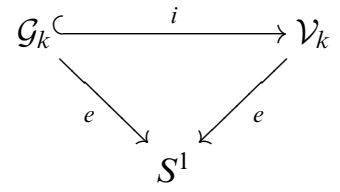

all maps represent isomorphisms in the category HTop.

Proof. For $k \neq 0$ it follows from Proposition 4.1 and Corollary 5.4 and for $k=0$ from Proposition 4.1 and Evaluation Lemma (Lemma 5.10).

\section{The proof of evaluation lemma.}

5.1. Retractions in mapping spaces. Consider a map $f:[0,2 \pi] \rightarrow \mathbb{R}$ such that $f(0)=f(2 \pi)$. We will make use of the following natural notation for functions

$$
f^{+}:=\max \{f, 0\}, \quad f^{-}:=\min \{f, 0\}
$$

and their integrals

$$
c^{+}(f):=\int_{0}^{2 \pi} f^{+} \mathrm{d} s, \quad c^{-}(f):=-\int_{0}^{2 \pi} f^{-} \mathrm{d} s, \quad c(f):=c^{+}(f)-c^{-}(f) .
$$


Recall that $\mathcal{V}_{k}=\left\{v: S^{1} \rightarrow \mathbb{R}^{2} \backslash\{0\} \mid \operatorname{deg} v=k\right\}$. By Proposition 3.1, $\mathcal{G}_{k}=\{v=(f, g) \in$ $\left.\mathcal{V}_{k} \mid c(f)=0\right\}$. Set

$$
\begin{aligned}
& \mathcal{V}_{k}^{\#}=\left\{v=(f, g) \in \mathcal{V}_{k} \mid c^{+}(f)>0 \text { and } c^{-}(f)>0\right\} \\
& \mathcal{G}_{k}^{\#}=\mathcal{G}_{k} \cap \mathcal{V}_{k}^{\#}
\end{aligned}
$$

LEMMA 5.1. For all $k, \mathcal{G}_{k}^{\#}$ is a strong deformation retract of $\mathcal{V}_{k}^{\#}$.

Proof. Consider the retraction $R: \mathcal{V}_{k}^{\#} \rightarrow \mathcal{G}_{k}^{\#}$ given by

$$
R(v)=R(f, g):=\left(\frac{\min \left\{c^{-}(f), c^{+}(f)\right\}}{c^{+}(f)} f^{+}+\frac{\min \left\{c^{-}(f), c^{+}(f)\right\}}{c^{-}(f)} f^{-}, g\right) .
$$

Observe that $i \circ R$ is homotopic to $\operatorname{Id}_{\mathcal{V}_{k}^{\#}}$ via the straight-line homotopy.

REMARK 5.2. In [5] Parusiński uses the similar retraction

$$
R(v)=R(f, g):=\left(\sqrt{\frac{c^{-}(f)}{c^{+}(f)}} f^{+}+\sqrt{\frac{c^{+}(f)}{c^{-}(f)}} f^{-}, g\right),
$$

which unfortunately does not work for our approach (see Lemma 5.6).

LemmA 5.3. For all $k \neq 0, \mathcal{V}_{k}=\mathcal{V}_{k}^{\#}$ and, in consequence, $\mathcal{G}_{k}=\mathcal{G}_{k}^{\#}$.

Proof. If $c^{+}(f)=0$ or $c^{-}(f)=0$, then $\operatorname{deg} v=0$, since in this case there is a straightline homotopy (with no zeros for $t \in(0,1])$ between $f$ and some non-zero constant function through $2 \pi$-periodic functions.

COROLlaRY 5.4. For each $k \neq 0$, the inclusion $\mathcal{G}_{k} \hookrightarrow \mathcal{V}_{k}$ is a homotopy equivalence.

REMARK 5.5. Observe that for $k=0$ we have $\mathcal{G}_{0} \not \subset \mathcal{V}_{0}^{\#}$ and $\mathcal{G}_{0}^{\#} \varsubsetneqq \mathcal{G}_{0}$ because two new non-empty contractible subspaces of $\mathcal{G}_{0}$ appear:

$$
\begin{aligned}
& \mathcal{G}_{0}^{+}:=\left\{v=(f, g) \in \mathcal{G}_{0} \mid f=0 \text { and } g>0\right\} \\
& \mathcal{G}_{0}^{-}:=\left\{v=(f, g) \in \mathcal{G}_{0} \mid f=0 \text { and } g<0\right\}
\end{aligned}
$$

Let us denote by $\mathcal{W}_{0}$ the subspace $\mathcal{V}_{0}^{\#} \cup \mathcal{G}_{0}^{-} \cup \mathcal{G}_{0}^{+}$. It is obvious that $\mathcal{W}_{0}=\{v=$ $\left.(f, g) \in \mathcal{V}_{0} \mid c^{+}(f)=0 \equiv c^{-}(f)=0\right\}$. In case $k=0$ the retraction from the proof of Lemma 5.1 can be easily extended from $\mathcal{G}_{0}$ to $\mathcal{W}_{0}$. By abuse of notation, we continue to write $R$ for this extension.

LEMMA 5.6. The map $R: \mathcal{W}_{0} \rightarrow \mathcal{G}_{0}$, given by

$$
R(v)= \begin{cases}\left(\frac{\min \left\{c^{-}(f), c^{+}(f)\right\}}{c^{+}(f)} f^{+}+\frac{\min \left\{c^{-}(f), c^{+}(f)\right\}}{c^{-}(f)} f^{-}, g\right) & \text { if } v \in \mathcal{V}_{0}^{\#}, \\ v & \text { if } v \in \mathcal{G}_{0}^{-} \cup \mathcal{G}_{0}^{+}\end{cases}
$$

is a strong deformation retraction. 


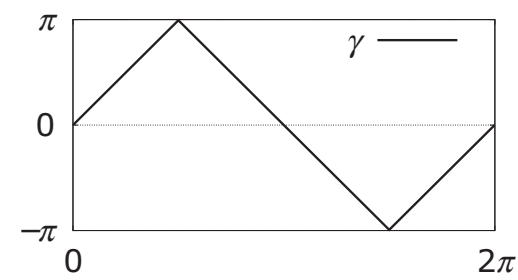

Figure 1. Function $\gamma$.

5.2. Homotopy equivalence between $\mathcal{G}_{0}$ and $S^{1}$. Let us denote by $p: \mathbb{R} \rightarrow[0,2 \pi]$ the natural projection $p(x):=x(\bmod 2 \pi)$. We will make use of an auxiliary function $\gamma:[0,2 \pi] \rightarrow \mathbb{R}$ given by

$$
\gamma(s):= \begin{cases}2 s & \text { if } s \in\left[0, \frac{\pi}{2}\right] \\ 2 \pi-2 s & \text { if } s \in\left[\frac{\pi}{2}, \frac{3 \pi}{2}\right] \\ 2 s-4 \pi & \text { if } s \in\left[\frac{3 \pi}{2}, 2 \pi\right]\end{cases}
$$

(see Figure 1) and its projection on $S^{1}$, i.e. $\gamma: S^{1} \rightarrow S^{1}, \gamma=p \circ \gamma$.

Observe that $(\boldsymbol{\gamma}, 1) \in \mathcal{G}_{0}$, since $\int_{0}^{2 \pi} \cos \gamma(s) \mathrm{d} s=0$.

The following definitions will be needed in the proof of Deformation Lemma (Lemma 5.8). Recall that a homotopy $g: X \times I \rightarrow X$ such that $g_{0}$ is the identity map is called a deformation of $X$ onto $Y:=\left\{g_{1}(x) \mid x \in X\right\}$. Furthermore, if $g_{1}(x)=x_{0}$ for all $x \in X$, then the deformation $g$ is called a contraction of $X$ to $x_{0}$. Note that for a homotopy $h: X \times I \rightarrow X$ and a deformation $g: X \times I \rightarrow X$ the 'composition of homotopies' $g * h: X \times I \rightarrow X$ given by

$$
(g * h)(x, t):= \begin{cases}h(x, 2 t) & \text { if } t \in\left[0, \frac{1}{2}\right] \\ g(h(x, 1), 2 t-1) & \text { if } s \in\left[\frac{1}{2}, 1\right]\end{cases}
$$

is well defined and continuous.

Let $A^{+}:=\{x \in \mathbb{R} \mid \cos x>0\}$ and $A^{-}:=\{x \in \mathbb{R} \mid \cos x<0\}$. We will denote by $\mathcal{L}$ the space of $2 \pi$-periodic continuous functions from $[0,2 \pi]$ to $\mathbb{R}$. We will also need the following subspaces of $\mathcal{L}$ :

$$
\begin{aligned}
\mathcal{L}^{a} & :=\{\varphi \in \mathcal{L} \mid \varphi(0)=a\}, \\
\mathcal{I} & :=\left\{\varphi \in \mathcal{L} \mid \operatorname{Im} \varphi \cap A^{+}=\emptyset \equiv \operatorname{Im} \varphi \cap A^{-}=\emptyset\right\}, \\
\Gamma & :=\{\gamma+a \in \mathcal{L} \mid a \in \mathbb{R}\} .
\end{aligned}
$$

By the definition of $\mathcal{I}$ and $\mathcal{W}_{0}$, we immediately obtain the following result.

Proposition 5.7. For any $r:[0,2 \pi] \rightarrow(0,+\infty)$,

$$
\varphi \in \mathcal{I} \equiv(p \circ \varphi, r) \in \mathcal{W}_{0}
$$

We finish this section with two key lemmas: Deformation Lemma and Evaluation Lemma.

Lemma 5.8 (Deformation Lemma). There is a deformation $h: \mathcal{L} \times I \rightarrow \mathcal{L}$ of $\mathcal{L}$ onto $\Gamma$ such that 
1. $h_{t}(\varphi+c)=h_{t}(\varphi)+c$ for all $t \in I$ and $c \in \mathbb{R}$,

2. $h_{1}\left(\mathcal{L}^{a}\right)=\{\gamma+a\}$ for all $a \in \mathbb{R}$,

3. $h_{t}(\mathcal{I}) \subset \mathcal{I}$ for all $t \in I$.

REMARK 5.9. Note that from (1), if $\psi=\varphi+2 k \pi$, then $h_{t}(\psi)=h_{t}(\varphi)+2 k \pi$ and, in consequence, $p \circ\left[h_{t}(\psi)\right]=p \circ\left[h_{t}(\varphi)\right]$. It may be worth pointing out that the deformation constructed in our proof also satisfies the following condition:

$$
h_{t}\left(\mathcal{L}^{a}\right) \subset \mathcal{L}^{a} \text { for all } t \in I \text { and } a \in \mathbb{R},
$$

but we will not use it here.

Proof. Let, for $\varphi \in \mathcal{L}$,

$$
m(\varphi):=\frac{\max \{|\varphi(s)| \mid s \in[0,2 \pi]\}}{\pi} .
$$

Consider a finite sequence $\left\{k^{i}\right\}_{i=1}^{5}$ of deformations $k^{i}: \mathcal{L}^{0} \times I \rightarrow \mathcal{L}^{0}$ given by the formulas

$$
\begin{aligned}
k^{1}(\varphi, t)(s) & := \begin{cases}\varphi\left(\frac{2 s}{2-t}\right) & \text { for } s \in[0,(2-t) \pi], \\
0 & \text { for } s \in[(2-t) \pi, 2 \pi],\end{cases} \\
k^{2}(\varphi, t)(s) & := \begin{cases}\varphi(s) & \text { for } s \in[0, \pi], \\
\varphi(s)+t m(\varphi) \gamma(s) & \text { for } s \in[\pi, 2 \pi],\end{cases} \\
k^{3}(\varphi, t) & :=(1-t) \varphi+t \max \{\varphi, m(\varphi) \gamma\}, \\
k^{4}(\varphi, t) & :=(1-t) \varphi+t m(\varphi) \gamma, \\
k^{5}(\varphi, t) & :=(1-t) \varphi+t \gamma .
\end{aligned}
$$

Let $k:=k^{4} *\left(k^{3} *\left(k^{2} * k^{1}\right)\right)$. By definition,

- $\operatorname{Im}\left(k_{t_{1}}(\varphi)\right) \subset \operatorname{Im}\left(k_{t_{2}}(\varphi)\right)$ for $0 \leq t_{1} \leq t_{2} \leq 1$,

- if $\varphi \equiv 0$, then $k_{t}(\varphi) \equiv 0$ for all $t \in I$.

Moreover, we emphasise that the above claim does not hold if we replace the deformation $k$ by any of the deformations $k^{4} * k^{1}, k^{4} * k^{2} * k^{1}$ etc. Let us define $K:=$ $k^{5} * k$. By the above, $K$ is a contraction of $\mathcal{L}^{0}$ to $\gamma$ such that for all $t \in I$

$$
\operatorname{Im} \varphi \subset \operatorname{Im}\left(K_{t}(\varphi)\right) \quad \text { or } \quad \operatorname{diam} \operatorname{Im}\left(K_{t}(\varphi)\right) \geq 2 \pi \text {. }
$$

Finally, we extend $K$ to the deformation $h: \mathcal{L} \times I \rightarrow \mathcal{L}$ by setting

$$
h(\varphi, t):=K(\varphi-\varphi(0), t)+\varphi(0) .
$$

It is easy to see that $h$ satisfies the conditions (1) -(3).

Lemma 5.10 (Evaluation Lemma). The evaluation map $e: \mathcal{G}_{0} \rightarrow S^{1}$, given by $e(v)=\theta(0)$ for $v=(\theta, r)$, is a homotopy equivalence. by

Proof. We show that the homotopical inverse of $e$ is the map $d: S^{1} \rightarrow \mathcal{G}_{0}$ defined

$$
d(\alpha)(s):=(\alpha+\gamma(s), 1)
$$




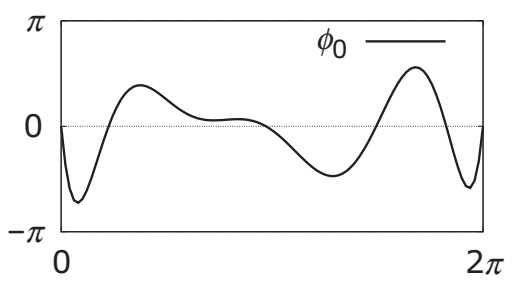

$$
\begin{aligned}
& \phi_{0}=k_{0}^{1}\left(\phi_{0}\right) \\
& \phi_{1}=k_{1}^{1}\left(\phi_{0}\right)
\end{aligned}
$$
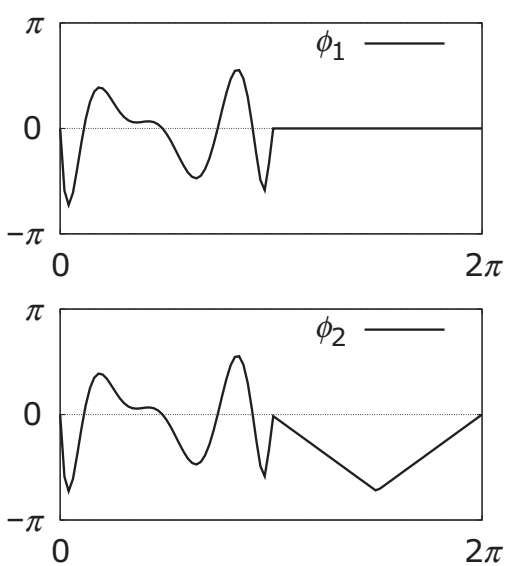

$$
\phi_{2}=k_{1}^{2}\left(\phi_{1}\right)
$$

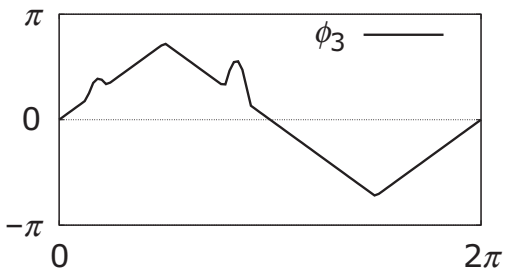

$$
\phi_{3}=k_{1}^{3}\left(\phi_{2}\right)
$$

$$
\phi_{4}=k_{1}^{4}\left(\phi_{3}\right)
$$

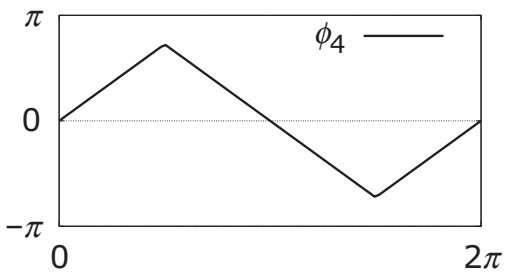

Figure 2. Deformation $k$ 'step by step.'

Precisely, since $e \circ d=\operatorname{Id}_{S^{1}}$, it is enough to show that the map $T:=d \circ e$ is homotopic to $\operatorname{Id}_{\mathcal{G}_{0}}$. Using the deformation $h$ from Deformation Lemma and the retraction $R$ from Lemma 5.6 we define the new deformation $H: \mathcal{G}_{0} \times I \rightarrow \mathcal{G}_{0}$ by setting

$$
H((\theta, r), t):=R\left(\Pi\left(p \circ\left[h_{t}(\varphi)\right],(1-t) r+t\right)\right),
$$

where $\varphi \in \mathcal{L}$ is any lift of $\theta$ (recall that $\Pi$ is transformation from polar to Cartesian coordinates). Observe that if $(\theta, r) \in \mathcal{G}_{0} \subset \mathcal{W}_{0}$, then $h_{t}(\varphi) \in \mathcal{L}$, in consequence, $\left(p \circ\left[h_{t}(\varphi)\right],(1-t) r+t\right)$ lies in the domain of the retraction $R$, i.e. the space $\mathcal{W}_{0}$. Moreover, $H_{0}=\operatorname{Id}_{\mathcal{G}_{0}}$ and $H_{1}=T$, where $T((\theta, r))=(\theta(0)+\gamma, 1)$. 
ACKNOWLEDGEMENTS. The authors want to express their thanks to the referee for several helpful comments concerning the paper.

\section{REFERENCES}

1. Z. Balanov, W. Krawcewicz, S. Rybicki and H. Steinlein, A short treatise on the equivariant degree theory and its applications, J. Fixed Point Theory Appl. 8 (2010), 1-74.

2. Z. Balanov, W. Krawcewicz and H. Steinlein, in Applied Equivariant Degree, (American Institute for Mathematical Sciences, Springfield, MO, 2006).

3. P. Bartłomiejczyk, K. Gęba and M. Izydorek, Otopy classes of equivariant local maps, J. Fixed Point Theory Appl. 25 (2010), 195-203.

4. E. N. Dancer, K. Gęba and S. Rybicki, Classification of homotopy classes of gradient equivariant maps, Fund. Math. 185 (2005), 1-18.

5. A. Parusiński, Gradient homotopies of gradient vector fields, Studia Math. XCVI (1990), 73-80. 